\title{
Numerical simulation of underwater acoustical field with directional sources based on the normal modes model
}

\author{
Wang Jihui*, Wang Zhenshan, Jia Bing \\ Science and Technology on Underwater Test and Control Laboratory, 116000, Dalian, China
}

\begin{abstract}
In this paper, beamforming and normal modes method are combined to calculate the underwater acoustical field. Firstly, beamforming technology is used to form directional sources by controlling the phase and relative amplitude. Secondly, considering the ocean speed, surface and bottom boundary, the point source sound field is calculated based on the normal modes model. Then underwater acoustical field with directional sources is achieved by adding all point source field. Taken the 11 elements vertical linear array as an example, the special directional source is formed by chebyshev amplitude weighting, and then underwater acoustical field is calculated in the Munk profile of different grazing angles. The results indicate that beam grazing angle is very essential to its sound field, and changing the grazing angle can enhance the sound intensity of the shadow zone correspondingly.
\end{abstract}

\section{Introduction}

The operating range of active sonar is decided by target characteristics and underwater sound channel in ocean environment. The propagation loss of active sonar signal can be derived with hydrology and bottom material parameters. In order to assess sonar performance, the operating range can be predicted combined with typical target scattering characteristics. Due to outstanding antireverberation ability, directional source is widely used in underwater active detection.

A beamformer is a spatial filter and can be used to increase the signal-to-noise ratio by blocking most of the noise outside the directions of interest. As practical engineering application, the signal phase is arranged at specific positions to enhance signal-to-reverberation ratio[ $[1,2]$. The sound field in complicated ocean channel by sonar beams propagation is the concerned problem in this thesis.

Acoustic Toolbox program is created by Maggi and Duncan of Curtin University of Technology in Perth, Australia. We can use the Acoustic Toolbox for the underwater environment modelling, which includes versions of BELLHOP, KRAKEN, and SCOOTER, etc. Users can select proper parameters of grazing angles for their simulation work[3-6]. However, optimization of the transmitting pattern function is weak. In order to design the transmitting acoustic beam in detail but not have the grazing angle only, acoustic propagation field simulation method is established based on vertical line array directional source and typical models are simulated in this paper.

\section{NUMERICAL METHODS}

Using the phase-shift beamforming technique, frequency is fed to the individual array element with the correct phase relationship so that it produces directional source in a desired direction.

\subsection{Phase-shift beamforming}

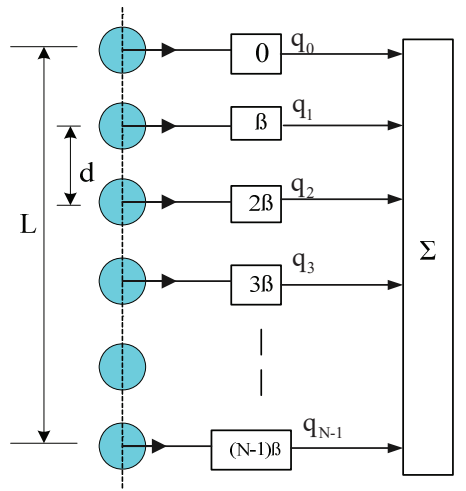

Figure 1. The structure of the perpendicular linear array.

Figure 1 shows the structure of the linear array. Each element is multiplied by the weights to form the array output signal[7]. Consider a linear array with $\mathrm{N}$ elements, $\mathrm{L}$ is the length, $\mathrm{d}$ is the spacing, the linear array with initial direction angle $\phi,(\mathrm{N}-1) \beta$ is compensation phase of each element and $q_{n}$ is corresponding weight function. Based on the mathematical model of a far-field plane

* Corresponding author: wangjihuihgc@ $163 . c 0 m$ 
wave, the transmitted signal is given by the following Equation 1 by adding the result of each element.

$$
\begin{aligned}
& R(\theta)=q_{0}+q_{1} \cos (\varphi-\beta)+q_{2} \cos (2(\varphi-\beta)) \\
& +\ldots q_{N-1} \cos ((N-1)(\varphi-\beta))
\end{aligned}
$$

The array factor $q_{i}$ is obtained using the method proposed by Dolph, which is based on the approximation to the Chebyshev polynomials of order $n$. The Chebyshev polynomials of order $n$ are defined as:

$$
\mathrm{T}_{\mathrm{n}}(x)=\cos (n \arccos x),|x| \leq 1
$$

While $x=\cos \phi$, and then Equation 3 becomes:

$$
T_{n}(x)=\cos (n \phi)
$$

It can generate a chebyshev-type amplitude distribution with a total number of elements $\mathrm{N}$ and the ratio of lobes[8].

\subsection{Normal modes method}

As actual ocean environments are usually complex, sound fields calculating is influenced by the factors including the sea surface roughness, typical SSP, layered media and so on. Normal modes method has been used for many years in underwater acoustics. On the assumption that the sound field is range independent, the normal modes method calculates an isolated point source in acoustic medium, which is based on separation of variables. The solution is governed by the acoustic helmholtz equation:

$$
\frac{1}{r} \frac{\partial}{\partial r}\left(r \frac{\partial p}{\partial r}\right)+\frac{\partial^{2} p}{\partial z^{2}}+\frac{\omega^{2}}{c^{2}(z)} p=-\frac{\delta(r) \delta\left(z-z_{s}\right)}{2 \pi r}
$$

Assume that the surface is pressure release boundary and the bottom at depth $\mathrm{D}$. is the perfect rigid boundary. The free surface condition is an approximation for the surface boundary condition. For the surface yields perfectly free boundary:

$$
p(0)=0
$$

The perfectly rigid assumption enjoys some popularity for a bottom boundary. For a purely acoustic problem, this becomes

$$
p^{\prime}(D)=0
$$

Solved by separation of variables, the pressure field is:

$$
p(r, z)=\frac{i}{4 \rho_{0}} \sum_{m=1}^{N} \psi_{m}\left(z_{s}\right) \psi_{m}(z) H_{0}^{(1)}\left(k_{r m} r\right)
$$

Where $r=\sqrt{\left(x_{P}-x_{Q}\right)^{2}+\left(y_{P}-y_{Q}\right)^{2}}, H_{0}^{(1)}$ is the Hankel function of the first kind of order zero. The equation has $N$ eigenvalues $k_{r m}$ and corresponding eigenvectors $\psi_{m}, z_{s}$ is the depth of the source[9].
Many ways can compute the equation numerically, and the finite difference method is chosen. The interval depth $0<z<D$ is divided into $\mathrm{N}$ equal intervals. Once the eigenvalues are found, the eigenvectors are computed using inverse iteration. The inverse iteration is given by equation 8 .

$$
A\left(k^{2}\right) Z=0
$$

Here, $A$ is a symmetric tridiagonal matrix defined, and $\mathrm{Z}$ is the vector with components $Z_{0}, Z_{1}, \ldots Z_{N}$. KRAKEN normal modes code in the Acoustic Toolbox is used in this thesis to model the sound field transmitted from the point source. KRAKEN normal mode model is robust, accurate, and efficient $[9,10]$. A version of KRAKEN with a MEX wrapper is used so that it can easily be called from Matlab software, and the Python Scripts source code is used for reading/writing input/output the sound field calculation results.

\section{Model simuliting and analysis}

\subsection{Computating model}

In the simulation, we consider classical chebyshev and uniform linear array of 11 elements with half wavelength spacing. The centre of the linear array is 1300 meters, the frequency value of the transmitted signal is $100 \mathrm{~Hz}$, and the ratio of lobes is $30 \mathrm{~dB}$. Figure 2 shows different simulation results using the uniform and chebyshev weights. We can observe that the peak side lobe with chebyshev weight is same $-30 \mathrm{~dB}$.

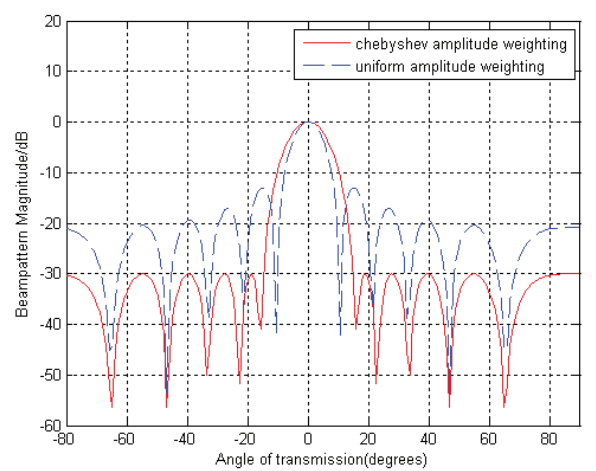

Figure 2. The rectangular plot of beam pattern magnitude of with uniform and chebyshev amplitude distribution.

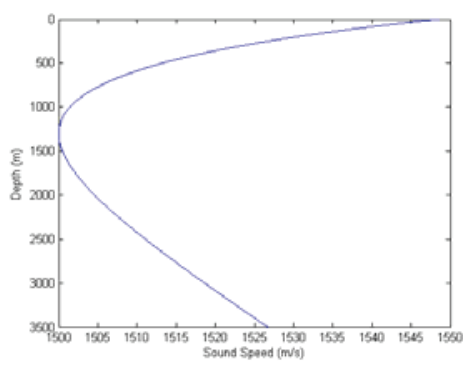

Figure 3. Munk profile. 
The Munk profile is an idealized sound speed profile but it can illustrate typical features of deep water SSP[11]. Consider the depth is 3500 meters, and the resulting profile is plotted in figure 3 .

\subsection{Sound field simulation}

\subsection{1 point source sound field simulation}

Figure 4 shows the selected modes 1, modes 20, modes 60 and modes 100 of the Munk profile with the surface pressure released and the bottom is perfect rigid boundary. Lower order modes in particular mode 1 is zero in the position near the sea surface and bottom, and all modes make contribute to the sound field.

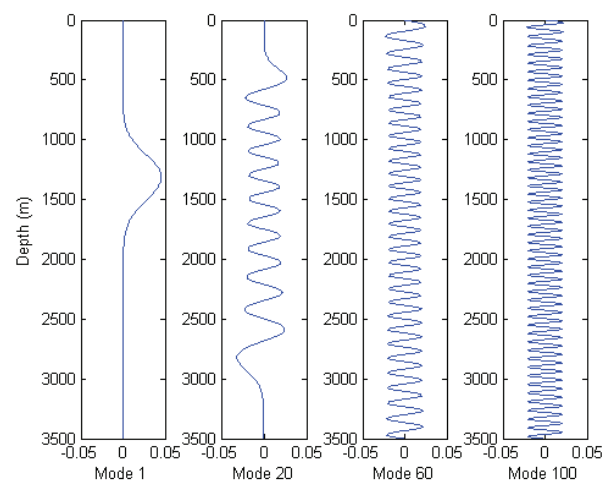

Figure 4. Selected modes in the Munk profile.

The transmission loss TL is calculated by Equation 9:

$$
T L=10 \log \frac{I(1)}{I(r)}
$$

Where $I(1), I(r)$ is the sound intensity at 1 meters and $\mathrm{r}$ meters far away from the equivalent sound center respectively.

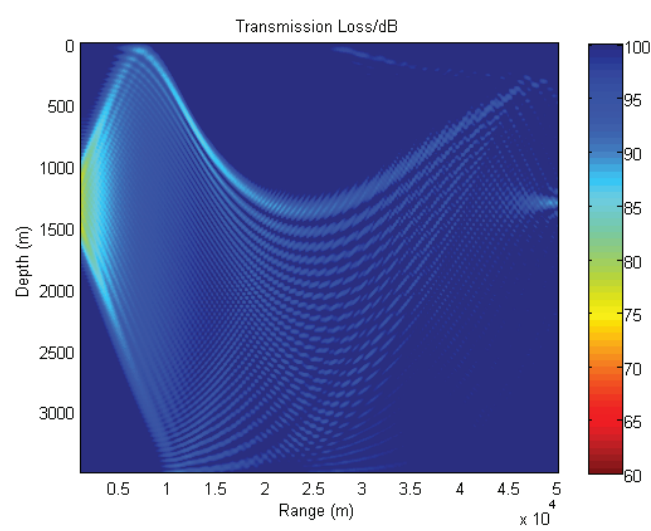

Figure 5. Horizontal range-depth sound field transmission loss distribution of point source.

Figure 5 shows the sound field transmission loss distribution generated by the point source. The horizontal range is $1-50$ kilometers, and the depth is from 0 to 3.5 kilometers. There is shadow zone clearly where nearly no sound wave reach.

\subsection{2 directional sources sound field simulation}

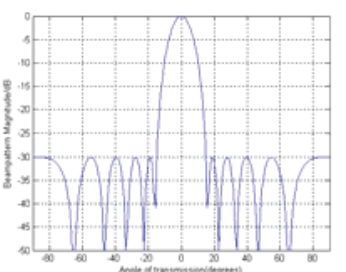

a.

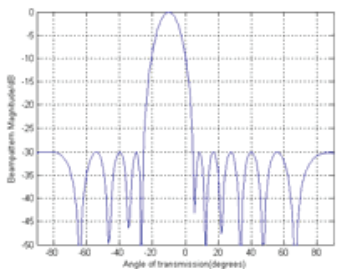

c.

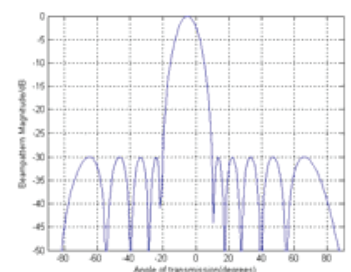

b.

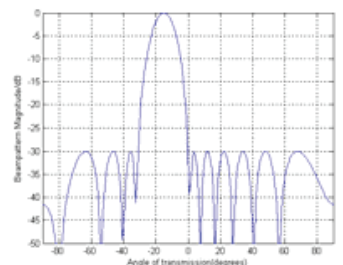

d.
Figure 6.Beam pattern magnitude of different direction angle is $0^{\circ}, 5^{\circ} 10^{\circ} 15^{\circ}$ in proper order a-d.

Figure 6 shows the beam pattern magnitude of different direction angles with chebyshev amplitude weight.

The point source sound field is calculated based on the normal modes model. Add the sound field generated by each point source and at last the underwater acoustical field with directional sources is achieved.

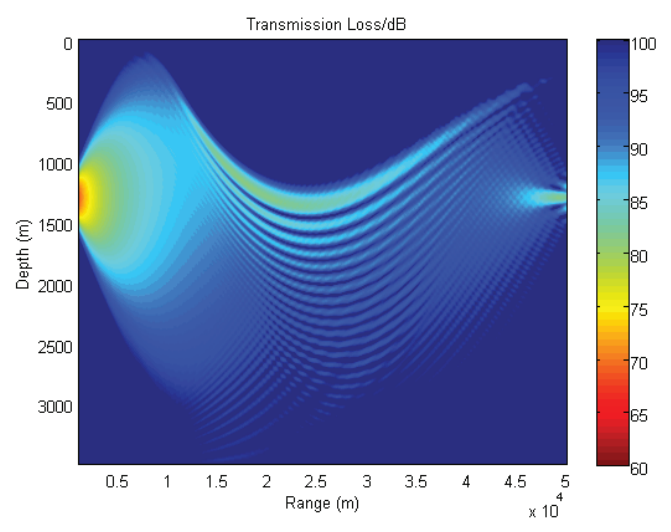

a. Grazing angle $0^{\circ}$

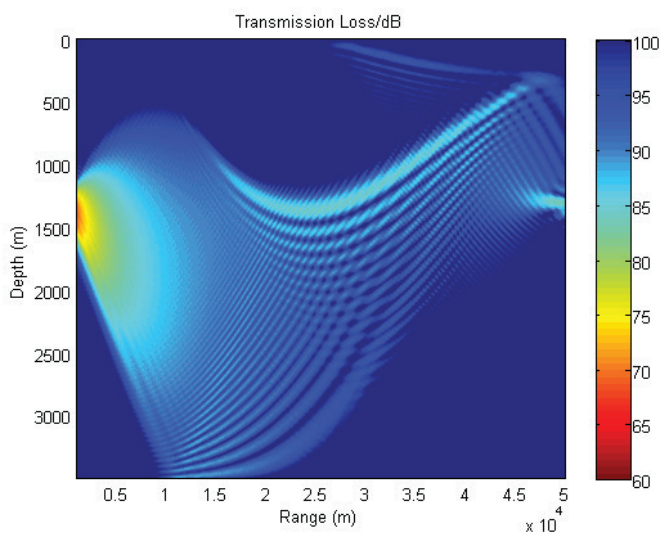

b. Grazing angle $5^{\circ}$ 


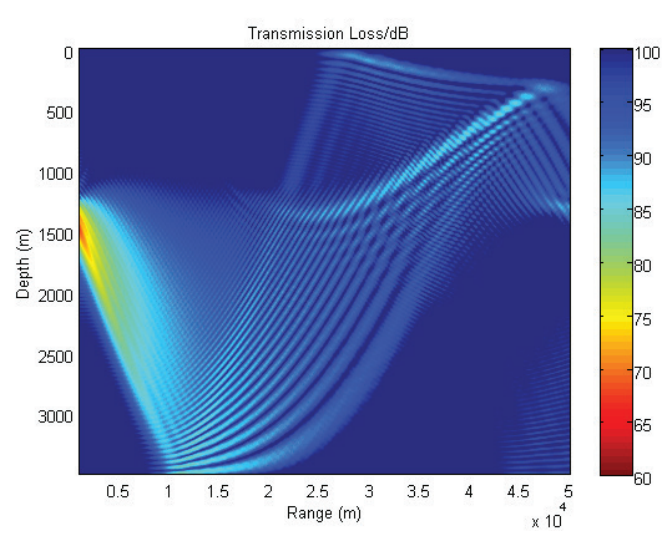

c. Grazing angle $10^{\circ}$

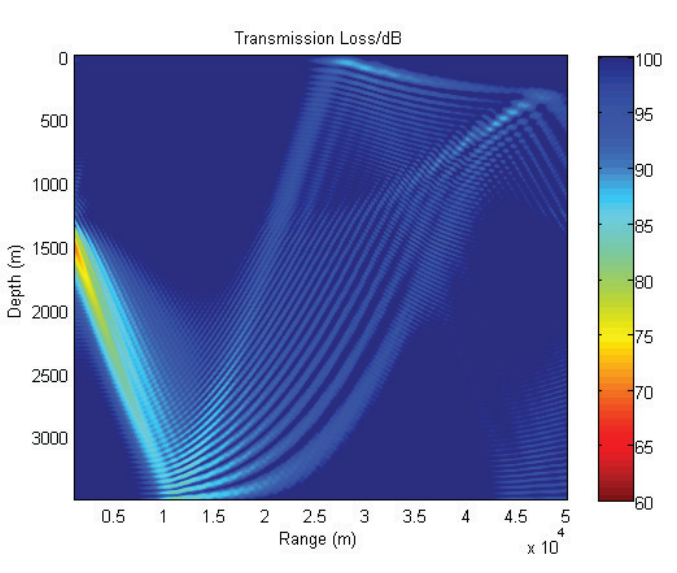

d. Grazing angle $15^{\circ}$

Figure 7. Horizontal range-depth transmission loss distribution of different grazing angles.

Figure 7 shows that the transmission loss has a convergence zone near the sound source, but the energy rapidly decline under the influence of the ocean sound speed profile and the reflection of the interface. Also it exists obvious shadow zone where the sound wave cannot reach.

There is the similar transmission loss distribution of grazing angle $0^{\circ}$ to the point source sound field when the grazing angle is low, see Figure $7 \mathrm{a}$. Contrasting on figure $7 \mathrm{~b}$, figure $7 \mathrm{c}$ and figure $7 \mathrm{~d}$, when the grazing angle increases, the energy of the ocean bottom reflection is stronger and stronger. It can reveal that the directional beam may enhance the sound intensity of the shadow zone for the reason that the sound wave can be reflected by the bottom.

\section{Conclusion}

Beamforming is spatial filtering, a means of transmitting sound preferentially in some directions over others. Instead of computing the sound field supported by the Acoustic Toolbox program directly, beamforming and KRAKEN normal modes method are combined to calculate the underwater acoustical field. This article provide a convenient method for designing the directional sources and researching its sound field generated in the oceanic waveguide.
Sound field of directional sources in deep sea has been investigated. The results indicate that it exists shadow zone where direct and reflect sound wave cannot reach, but the sound intensity can be enhanced by varying the grazing angle of the directional sources. If the target places in the shadow zone, the traditional method has many difficulties, but this method can be probably helpful for underwater target detection and may have some significance for active sonar system.

\section{References}

1. M A Ainslie, Principles of Sonar Performance Modeling, Springer (2010)

2. J. Benesty, J. Chen, and Y. Huang, Microphone Array Signal Processing, M (2008)

3. Mieheal B. Porter. The BELLHOP Manual and User's Guide:PRELIMINARY DRAFT.Heat, Light, and Sound Research, Inc(2010)

4. M.B.Porter.(2011,Jan.).The BELLHOP manual and user's guide:PRELIMINARY DRAFT. Heat, Light, and Sound Research,Inc (2012)

5. DA Lianglong, Modeling and Application of Underwater Acoustic Environmental Effect[M]. Beijing, Science Press(2012)

6. Alec J Duncan, Iain M Parnum and Peter J Henley. Efficient modeling of mid to high frequency underwater acoustic propagation[J].Proceedings of Acoustics(2013)

7. Shafqat U K, Ijaz M Q, Bial S and Aqdas N, Recovery of failed element signal with a digitally beamforming using linear Symmetrical array antenna, J. Journal of information science and engineering. 32 (2016)

8. R. E. Blabut, W. Millerm, and C. H. Wilcox, Radar and Sonar, Part I, Springer-Verlag, New York(1991)

9. Jensen F B, Kuperman W A, Porter M B, Schmidt Henrik, Computational Ocean Acoustics(2011)

10. L.M. Brekhovskikh and Yu P. Lysanov, Fundamentals of Ocean Acoustics, SpringerVerlag (2003)

11. W.H.Munk, Sound channel in an exponentially stratified ocean with applications to SOFAR, J. Acoust. Soc. Am. 55(1974) 